\title{
DE
}

DE GRUYTER

OPEN

BULGARIAN ACADEMY OF SCIENCES

CYBERNETICS AND INFORMATION TECHNOLOGIES • Volume 14, Special Issue

Sofia $\bullet 2014$

Print ISSN: 1311-9702; Online ISSN: 1314-4081

DOI: $10.2478 /$ cait-2014-0039

\section{Research Design of an Adaptive Controller Based on Desired Trajectory Compensation of Neural Networks}

\author{
Liu Yu \\ School of Computer Engineering, Jinling Institute of Technology, Nanjing 211169 China \\ Email:ayu987@jit.edu.cn
}

\begin{abstract}
This paper has designed a variable structure controller based on the nominal compensation of neural networks. The neural network input is the desired trajectory, which eliminates the strict assumptions of the control inputs in conventional neural networks. It also ensures the asymptotic stability of the system closed-loop global exponentials to introduce model compensation and continuous variable structure control rate. By means of Lyapunov stability theory, it is analyzed and researched how to guarantee good transient performance of the control system comprehensively and thoroughly. The theoretic analysis and simulation results demonstrate the efficiency of the method proposed.
\end{abstract}

Keywords: Neural networks, robot, tracking control, trajectory compensation, adaptive controller.

\section{Introduction}

As intelligent control theory has been paid more and more attention in control fields, many scholars have applied it to uncertainty robot system control. The fuzzy control has better reasoning ability. For example, in [1] a class of robust control strategy based on fuzzy control is designed. It mainly uses the reasoning ability of fuzzy control to determine the limits of uncertainty parts. The conventional control strategy is dependent on the model, while the neural networks have good nonlinear function approximation ability, which can be used in a robot system control to achieve a better control effect. The self-adaptation control method of neural networks provides a new way to study complex nonlinear control problems. Moreover, self-adaptation control of an uncertain nonlinear system based on neural 
networks has become one of the hot topics in the study of control theory, and many research results have been made [2-5].

The authors present in [6] a typical robot control method based on a neural network, which integrates the neural network method and the variable structure control to develop a new control strategy. It uses the neural network method to approach the unknown nonlinear model of the system, and makes use of the variable structure control to compensate the approximation error of the model that guarantees the robustness of the system.

In $[7,8]$ the authors adopt GGAP-RBF (growing and pruning RBF) algorithm based on the concepts of neuron sensitivity and winning neurons to ensure the structure and initial parameters of the neural networks online. Because every time it is only needed to adjust the parameters of the near neuron, the amount of calculation is greatly reduced, so that the speed is improved. The number of hidden layer neurons starts from a scratch; the algorithm has the standards of increasing and deleting nodes; therefore, it can obtain a more compact network structure.

$\mathrm{Horng}$ and $\mathrm{Hs}$ iseh [9] utilize CMAC to gain from the uncertainty parts of a control system to realize stable and efficient control of the closed loop control system. However, this kind of control strategy requires the assumption, that the state of the system is bounded, and the neural network with the adaptive control method only learns the network weights online; there is nothing it can do for the model approximation error and the existing unavoidable nonlinear external interferences in the system. In [10] the NDOFEL method for time-varying nonlinear systems with unknown interference is proposed, combining the nonlinear disturbance observer and the neural network strategy based on FEL. It improves the tracking precision and solves the problem that the observation error cannot converge to zero when $\dot{d}(t) \neq 0$. Reference [11] presents a neural network disturbance observer method for a class of multi-input and multi-output system. The authors in $[12,13]$ make use of the variable structure control items to compensate the model approximation errors and external disturbances. But it cannot guarantee the transient performance of the system well, and the existence of discontinuous terms in the control algorithm will cause a chattering phenomenon of the system.

Aiming at the shortage that conventional robust control methods can keep the system stable, but do not guarantee a good transient performance, the paper suggests in the model compensation to design a variable structure controller based on neural network nominal compensation. And through Lyapunov method it improves the update rate of the traditional network weights appropriately to make the practical motion trajectory of the robot system track the desired trajectory rapidly according to the index law; and the trajectory tracking errors are uniformly bounded ultimately. In this process the system not only ensures good dynamic performance, but it can also still be robust in the presence of external disturbances. At the same time, based on Lyapunov stability theory, the research results of this paper are proved rigorously and theoretically. The contrast of the simulation experiments verifies the advanced and efficient nature of the control strategy. The control strategy proposed can be used as an important reference for similar complex nonlinear mechanical system control. 


\section{Model compensation}

The mathematical model of robots is usually described by the following equation

$$
M(q) \ddot{q}+C(q, \dot{q}) \dot{q}+G(q)+F(\dot{q}, q)+d(t)=\tau .
$$

In that equation $q, \dot{q}, \ddot{q} \in R^{n}$ are respectively expressed as the joint position vector, velocity vector and acceleration vector; $M(q) \in R^{n \times n}$ is the inertia matrix of the robot arms; $C(q, \dot{q}) \in R^{n \times n}$ denotes Coriolis force or Centrifugal force matrix of the robot arm; $G(q) \in R^{n}$ is the gravity item of the robot; $d(t)$ is the external random disturbance; $F(\dot{q}, q) \in R^{n}$ expresses the friction item. The nonlinear friction $F(\dot{q}, v)$ can be indicated as $F(\dot{q}, q)=F_{\mathrm{s}} \dot{q}+F_{\mathrm{d}}(q)$, where $F_{\mathrm{s}}, F_{\mathrm{d}}(q)$ are respectively shown as an unknown static friction item and an dynamic friction item.

The definition of the nominal model of the system is as follows:

$$
M\left(q_{d}\right) \ddot{q}_{d}+C\left(q_{d}, \dot{q}_{d}\right) \dot{q}_{d}+G\left(q_{d}\right)+F\left(\dot{q}_{d}, q_{d}\right)=\tau_{d},
$$

where $q_{d}, \dot{q}_{d}, \ddot{q}_{d} \in R^{n}$ are respectively expressed as an expectation joint position vector, velocity vector and acceleration vector. We assume that the boundary of $d(t)$, the unstructured random disturbance is known, and $d(t) \leq d_{M} \forall t \in R^{+}$.

Control objective: Define the target trajectory as $\ddot{q}_{d}, \dot{q}_{d}, q_{d} \in R^{n}$ and $q_{d}$ are second derivatives sectionally continuous. The control objective of this paper is to look for a control strategy $\tau$ to make the actual motion trajectory track the target trajectory. Meanwhile, ensure that $\dot{e}$ and $e$ are exponential asymptotic or exponential bounded stability in the global scope.

We will design a variable structure controller based on a special sliding model. Define the model compensation as:

$$
\left\{\begin{array}{lccc}
\dot{z}=A_{z} z+B_{z} e & z \in R^{n_{z}} & A_{z} \in R^{n_{z} \times n_{z}} & B_{z} \in R^{n_{z} \times n} ; \\
y_{z}=C_{z} z+D_{z} e & y \in R^{n} & C_{z} \in R^{n \times n_{z}} & D_{z} \in R^{n \times n} .
\end{array}\right.
$$

The expected response of the sliding mode process can be ensured by means of the proper selection of matrix $\left(A_{z}, B_{z}, C_{z}, D_{z}\right)$.

We set

$$
r=\dot{e}+y_{z}=\dot{q}-\dot{q}_{r}, \quad \dot{q}_{r}=\dot{q}_{d}(t)-y_{z} .
$$

Thus we can get the relationship between $r$ and $e$ :

$$
e=G_{r}^{-1}(s) r \text {, }
$$

where

$$
\begin{gathered}
G_{r}(s)=s I_{n}+G_{z}(s), \\
G_{z}(s)=C_{z}\left(s I_{n z}-A_{z}\right)^{-1} B_{z}+D_{z} .
\end{gathered}
$$


Formula (5) is described by state spaces as

$$
\dot{x}_{r}=A_{r} x_{r}+B_{r} r, \quad y_{r}=C_{r} x_{r},
$$

where

$$
x_{r}=\left[z^{\mathrm{T}}, e^{\mathrm{T}}\right]^{\mathrm{T}} \in R^{n_{z}+n}, A_{r}=\left[\begin{array}{cc}
A_{z} & B_{z} \\
C_{z} & D_{z}
\end{array}\right], B_{r}=\left[\begin{array}{l}
0 \\
I_{n}
\end{array}\right], C_{r}=\left[\begin{array}{ll}
0 & I_{n}
\end{array}\right] .
$$

According to (5), in the sliding mode, the response of the system is controlled by $G_{r}^{-1}(s)$. As long as $G_{r}^{-1}(s)$ is stable, the sliding mode of the system will be stable. $G_{r}^{-1}(s)$ can be designed through the selection of $G_{r}(s)$ properly, that is, reach the expected dynamic responses in the sliding mode by selecting appropriate model compensation. The sliding mode can converge at an arbitrary exponential rate.

The above mentioned is equivalent to the state in the state space, i.e., according to (6) and Lyapunov stability theory, for any given positive definite matrix $Q$, there is only one real symmetric positive definite matrix $P$ to satisfy the following Lyapunov equation:

$$
A_{r}^{\mathrm{T}} P+P A_{r}=-Q \text {. }
$$

Then, through a proper design providing that the poles of $A_{r}$ are in the left plane and are away from $y$ axis, we can randomly design $\frac{\lambda_{\min }(Q)}{\lambda_{\min }(P)}$. So we can get the arbitrary exponential convergence speed.

Placing (2) and (4) into (1), we get:

$$
M(q) \dot{r}+C(q, \dot{q}) r+\Delta w\left(s, x_{r}\right)+d(t)=\tau-\tau_{d},
$$

where

$$
\begin{aligned}
\Delta w\left(r, x_{r}\right)= & \left(M(q) \ddot{q}_{r}-M\left(q_{d}\right) \ddot{q}_{d}\right)+\left(C(q, \dot{q}) \dot{q}_{r}-C\left(q_{d}, \dot{q}_{d}\right) \dot{q}_{d}\right)+ \\
& +\left(G(q)-G\left(q_{q}\right)\right)+\left(F(q, \dot{q})-F\left(q_{d}, \dot{q}_{d}\right)\right) .
\end{aligned}
$$

By the results in [14] we know the following equation:

$$
\Delta w\left(r, x_{r}\right) \leq \gamma_{1}\left\|x_{r}\right\|+\gamma_{2}\|r\|+\gamma_{3}\|r\|\left\|x_{r}\right\|+\gamma_{4}\left\|x_{r}\right\|^{2} .
$$

\section{Design of the controler}

The ideology for a nominal model $\tau_{d}=f(x)$ is also similar to that in [15]. Using the linear parametric network RBF, the local generalization network, to approach it in order to greatly accelerate the speed of learning.for the nonlinear model. 
The input vector of the network is $x=\left(q_{d}, \dot{q}_{d}, \ddot{q}_{d}\right)$. The radial basis function $\phi(x)$ is a Gaussian function, namely, $\phi_{j}=\exp \left(-\frac{\left\|x-c_{j}\right\|^{2}}{\sigma_{j}^{2}}\right)$, in which $c_{j}$ is the width of the odd function.

Based on the function approximation ability of RBF networks, we assume that: for any arbitrarily small positive $\varepsilon_{M}$, the largest weight vector $\theta^{*}$ can be always found, and thus to approach the approximation error $|\varepsilon(t)|=\left|\theta^{* \mathrm{~T}} \phi(x)-f(x)\right|<\varepsilon_{M}$. The optimal weights are bounded, i.e., there is a positive constant $\lambda$ satisfying $\left\|\theta^{*}\right\| \leq \lambda$.

Then the neural network approximation of the system nominal model can be expressed as $\hat{\tau}_{d}=\hat{\theta}^{\mathrm{T}} \phi\left(q_{d}, \dot{q}_{d}, \ddot{q}_{d}\right) ; \hat{\tau}_{d}$ is the estimation output of networks; $\hat{\theta}=\left(\hat{\theta}_{1}, \cdots, \hat{\theta}_{n}\right)$ is the estimation weight coefficient matrix; $\phi\left(q_{d}, \dot{q}_{d}, \ddot{q}_{d}\right)$ is the multi-variable function vector and it could be written as

$$
\tau_{d}=\theta^{* \mathrm{~T}} \phi\left(q_{d}, \dot{q}_{d}, \ddot{q}_{d}\right)+\varepsilon(t) .
$$

The input of the neural network is the desired trajectory, which is generally definite and bounded. Thus the routine bounded assumption of the input in neural networks is eliminated.

Then the control rate design of uncertain robot system is as follows:

$$
\begin{aligned}
& \tau=\hat{\theta}^{\mathrm{T}} \phi\left(q_{d}, \dot{q}_{d}, \ddot{q}_{d}\right)-K_{r} r-K_{x} x_{r}-r_{5}\left\|x_{r}\right\|^{2} r+\tau^{\prime}, \\
& \tau^{\prime}=\left\{\begin{array}{l}
-K_{\tau} r,\|r\| \leq \varphi_{d}, \quad \varphi_{d} \cong \frac{\varphi(t)}{K_{d}+\varepsilon_{1}}, \\
-\left(1-c_{1}\right) K_{\tau} r-c_{1} K_{d} \frac{r}{\|r\|}, \varphi_{d} \leq\|r\| \leq\left(1+\varepsilon_{2}\right) \varphi_{d}, \\
-K_{d} \frac{r}{\|r\|}, \quad\|r\| \geq\left(1+\varepsilon_{2}\right) \varphi_{d},
\end{array}\right.
\end{aligned}
$$

where $\hat{\theta}$ is the estimated value of $\theta ; K_{r}$ and $K_{x}$ are gain matrices, and $K_{x}=P B_{x}$; $r_{5}$ is the undetermined coefficient; $\tau^{\prime}$ is the smooth variable structure compensator in order to remove the network approximation error and external disturbances; $K_{d}=\varepsilon_{M}+d_{M} ; K_{\tau}$ is an arbitrary positive definite matrix; $c_{1}=\frac{\|r\|-\varphi_{d}}{\varepsilon_{2} \varphi_{d}} ; \varepsilon_{1}$ and $\varepsilon_{2}$ are arbitrary positives; $\varphi(t)$ is a bounded positive function, at that $0 \leq \varphi(t) \leq \varphi_{\max }$. 
Note that the above variable structure control part $\tau^{\prime}$ is similar to the idea in [14]. Introducing the boundary layer $\varphi(t)$ near to the sliding mode $\{\|r\|=0\}$ makes the variable structure control rate continuous that efficiently avoids the chattering problem of the system.

The weight adaptive rate is

$$
\hat{\theta}=-\Gamma \phi\left(q_{d}, \dot{q}_{d}, \ddot{\mathrm{q}}_{d}\right) r-\Gamma W \hat{\theta},
$$

where:

$$
\hat{\theta}=\hat{\theta}-\theta, \quad W=\left\{\begin{array}{l}
0, \quad\left\|\Gamma^{\prime}\left(\hat{\theta}-\theta_{a}\right)\right\| \leq \delta, \\
\rho \frac{\left\|\Gamma^{\prime}\left(\hat{\theta}-\theta_{a}\right)\right\|-\delta}{\varepsilon_{4}} \Gamma^{\prime^{2}}\left(\hat{\theta}-\theta_{a}\right), \\
\delta \leq\left\|\Gamma^{\prime}\left(\hat{\theta}-\theta_{a}\right)\right\| \leq \delta+\varepsilon_{4}, \\
\rho \Gamma^{\prime 2}\left(\hat{\theta}-\theta_{a}\right), \quad\left\|\Gamma^{\prime}\left(\hat{\theta}-\theta_{a}\right)\right\| \geq \delta+\varepsilon_{4} ;
\end{array}\right.
$$

both $\Gamma$ and $\Gamma^{\prime}$ are positive definite matrices which are known apriori; $\theta_{a}$ is a nominal amount; $\delta$ is a given positive value; $\rho$ and $\varepsilon_{4}$ are arbitrary positives.

Theorem 1. For the robot system described in (1), satisfying the assumption conditions, using the control rate shown in (11) and the weight adaptive rate of the neural networks shown in (12) makes the whole robot system meet the state that the global exponential is of an asymptotic consistency and bounded stability.

P r o of: Define Lyapunov function as

$$
V=\frac{1}{2} r^{\mathrm{T}} M(q) r+\frac{1}{2} x_{r}^{\mathrm{T}} P x_{r}+\frac{1}{2} \tilde{\theta}^{\mathrm{T}} \Gamma^{-1} \tilde{\theta} .
$$

The results are:

$$
\begin{aligned}
& \frac{1}{2} k_{1}\|r\|^{2}+\frac{1}{2} \lambda_{\min }(P)\left\|x_{r}\right\|^{2}+\frac{1}{2} \lambda_{\min }\left(\Gamma^{-1}\right)\|\tilde{\theta}\|^{2} \leq V \leq \\
& \leq \frac{1}{2} k_{2}\|r\|^{2}+\frac{1}{2} \lambda_{\max }(P)\left\|x_{r}\right\|^{2}+\frac{1}{2} \lambda_{\max }\left(\Gamma^{-1}\right)\|\tilde{\theta}\|^{2} .
\end{aligned}
$$

Take the derivative of (13) and put it into (8). Then we get

$$
\begin{gathered}
\dot{V}=r^{\mathrm{T}}\left[\tau-\tau_{d}-\Delta w\left(r, x_{r}\right)-d(t)\right]-\frac{1}{2} x_{r}^{\mathrm{T}} Q x_{r}+ \\
+x_{r}^{\mathrm{T}} P B_{r} r+\tilde{\theta}^{\mathrm{T}} \Gamma^{-1} \dot{\tilde{\theta}} .
\end{gathered}
$$

Substitute it into (10) and the control rate (11); by $K_{x}=P B_{r}$ we can get 


$$
\begin{gathered}
\dot{V}=r^{\mathrm{T}}\left[\tilde{\theta} \phi\left(q_{d}, \dot{q}_{d}, \ddot{q}_{d}\right)-K_{r} r-\Delta w\left(r, x_{r}\right)-\gamma_{5}\left\|x_{r}\right\|^{2} r+\tau^{\prime}-\varepsilon(t)-d(t)\right]- \\
-\frac{1}{2} x_{r}^{\mathrm{T}} Q x_{r}+\tilde{\theta}^{\mathrm{T}} \Gamma^{-1} \dot{\tilde{\theta}},
\end{gathered}
$$

where $\tilde{\theta}=\hat{\theta}-\theta^{*}$ expresses the estimation error of the optimal weight vector.

Substituting into the weight adaptive rate equation (12) we get

$$
\begin{gathered}
\dot{V}=r^{\mathrm{T}}\left[-K_{r} r-\Delta w\left(r, x_{r}\right)-\gamma_{5}\left\|x_{r}\right\|^{2} r+\tau^{\prime}-\varepsilon(t)-d(t)\right]- \\
-\frac{1}{2} x_{r}^{\mathrm{T}} Q x_{r}-\tilde{\theta}^{\mathrm{T}} W \hat{\theta} \\
\dot{V} \leq-r^{\mathrm{T}} K_{r} r+\gamma_{1}\left\|x_{r}\right\|\|r\|+\gamma_{2}\|r\|^{2}+\gamma_{3}\|r\|^{2}\left\|x_{r}\right\|+ \\
+\gamma_{4}\left\|x_{r}\right\|^{2}\|r\|-\gamma_{5}\left\|x_{r}\right\|^{2}\|r\|^{2}+r^{T}\left[\tau^{\prime}-\varepsilon(t)-d(t)\right]- \\
-\frac{1}{2} x_{r}^{\mathrm{T}} Q x_{r}-\tilde{\theta}^{\mathrm{T}} W \hat{\theta} .
\end{gathered}
$$

On the basis of the inequation $\beta_{1}|a||b| \leq \beta_{2}|a|+\beta_{3}|b|$, where $\forall a, b \in R, \quad \beta_{1}, \beta_{2}, \beta_{3} \geq 0, \beta_{1}^{2}=4 \beta_{2} \beta_{3}$, then

$$
\begin{gathered}
\dot{V} \leq-r^{\mathrm{T}} K_{r} r+\gamma_{6}\|r\|^{2}+\gamma_{7}\|r\|^{2}+\gamma_{2}\|r\|^{2}+\gamma_{8}\|r\|^{2}\left\|x_{r}\right\|^{2}+ \\
+\gamma_{9}\|r\|^{2}+\gamma_{10}\|r\|^{2}\left\|x_{r}\right\|^{2}+\gamma_{11}\left\|x_{r}\right\|^{2}-\gamma_{5}\left\|x_{r}\right\|^{2}\|r\|^{2}+ \\
+r^{\mathrm{T}}\left[\tau^{\prime}-\varepsilon(t)-d(t)\right]-\frac{1}{2} x_{r}^{\mathrm{T}} Q x_{r}-\tilde{\theta}^{\mathrm{T}} W \hat{\theta}= \\
=-r^{\mathrm{T}}\left[K_{r}-\left(\gamma_{6}+\gamma_{2}+\gamma_{9}\right)\right] r-\frac{1}{2} x_{r}^{\mathrm{T}}\left[Q-\left(\gamma_{7}+\gamma_{11}\right)\right] x_{r}- \\
-\left[\gamma_{5}-\left(\gamma_{8}+\gamma_{10}\right)\right]\|r\|^{2}\left\|x_{r}\right\|^{2}+r^{\mathrm{T}}\left[\tau^{\prime}-\varepsilon(t)-d(t)\right]-\tilde{\theta}^{\mathrm{T}} W \hat{\theta},
\end{gathered}
$$

where $\gamma_{6} \gamma_{7}=\frac{1}{4} \gamma_{1}^{2}, \quad \gamma_{8} \gamma_{9}=\frac{1}{4} \gamma_{3}^{2}, \gamma_{10} \gamma_{11}=\frac{1}{4} \gamma_{4}^{2}$.

Set $K_{r}, Q, \gamma_{5}$ appropriately and make it satisfy the following equations:

$$
\begin{gathered}
\lambda_{\text {min }}\left(K_{r}\right) \geq \varepsilon_{3}+\gamma_{6}+\gamma_{2}+\gamma_{9}, \\
\lambda_{\text {min }}(Q) \geq 2\left(\varepsilon_{3}+\gamma_{7}+\gamma_{11}\right), \\
\gamma_{5} \geq \gamma_{8}+\gamma_{10},
\end{gathered}
$$

where $\varepsilon_{3}$ is an arbitrary positive.

For $\quad r^{\mathrm{T}}\left[\tau^{\prime}-\varepsilon(t)-d(t)\right], \quad$ when $\quad\|r\| \geq\left(1+\varepsilon_{2}\right) \varphi_{d}, \quad$ then $r^{\mathrm{T}}\left[\tau^{\prime}-\varepsilon(t)-d(t)\right] \leq-K_{d}\|r\|+\left(\varepsilon_{M}+d_{M}\right)\|r\|=0$. When $\|r\| \leq\left(1+\varepsilon_{2}\right) \varphi_{d}$, then 
$K_{d}\|r\| \leq\left(1+\varepsilon_{2}\right) K_{d} \varphi_{d} \leq\left(1+\varepsilon_{2}\right) \varphi_{t}, r^{\mathrm{T}} \tau^{\prime} \leq 0$. Thus we get:

$$
\begin{gathered}
r^{\mathrm{T}}\left[\tau^{\prime}-\varepsilon(t)-d(t)\right] \leq\left(\varepsilon_{M}+d_{M}\right)\|r\|+r^{\mathrm{T}} \tau^{\prime}= \\
=K_{d}\|r\|+r^{\mathrm{T}} \tau^{\prime} \leq\left(1+\varepsilon_{2}\right) \varphi(t) .
\end{gathered}
$$

For $\tilde{\theta}^{\mathrm{T}} W \hat{\theta}$, put into $W$ we get:

$$
\tilde{\theta}^{\mathrm{T}} W \hat{\theta} \geq k_{1}\|\tilde{\theta}\|^{2}-k_{2},
$$

where $k_{1}=\left(\rho-\rho^{\prime}\right) \lambda_{\min }^{2}\left(\Gamma^{\prime}\right), k_{2}=\max \left\{\frac{\rho^{2} \delta^{2}}{4 \rho^{\prime}},\left(\rho-\rho^{\prime}\right)\left(2 \delta+\varepsilon_{4}\right)^{2}\right\}$, in which $\rho^{\prime}$ is a constant, and satisfies the condition $0<\rho^{\prime}<\rho$.

The integrated formulas (15), (16) and (17) are shown.

$$
\dot{V} \leq-\varepsilon_{3}\left(\|r\|^{2}+\left\|x_{r}\right\|^{2}\right)-k_{1}\|\tilde{\theta}\|^{2}+k_{2}+\left(1+\varepsilon_{2}\right) \varphi(t) .
$$

Summing up the above, we get

$$
\dot{V} \leq-\mu V+\left[k_{2}+\left(1+\varepsilon_{2}\right) \varphi_{\max }\right],
$$

where $\mu=\frac{2 \min \left(\varepsilon_{3}, k_{1}\right)}{\max \left(k_{2} \lambda_{\max }(P), \lambda_{\max }\left(\Gamma^{-1}\right)\right)}$.

Thus

$$
V \leq e^{-\mu t} V(0)+\frac{k_{2}+\left(1+\varepsilon_{2}\right) \varphi_{\max }}{\mu}\left(1-e^{-\mu t}\right) .
$$

Based on Lyapunov stability theory, we know that the whole closed-loop control system is stable, at the same time, the trajectory tracking errors are a uniformly bounded convergent, and by the exponential convergence stability theorem, we also know that the system meets the exponential convergence. Finally the theorem is proved.

The controller structure is shown in Fig. 1.

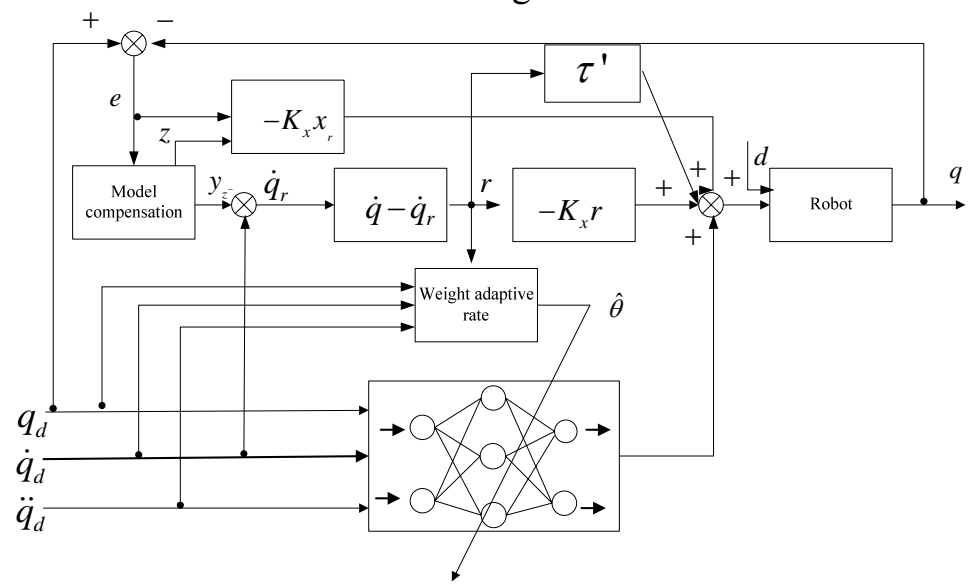

Fig. 1. A nominal compensation control system structure for a robot based on a neural network 


\section{Simulation and analysis}

In order to verify the efficiency of this control algorithm, we carry on the simulation of the two-degrees of a freedom mechanical arm system [16, 17]. The math model diagram is shown in Fig. 2.

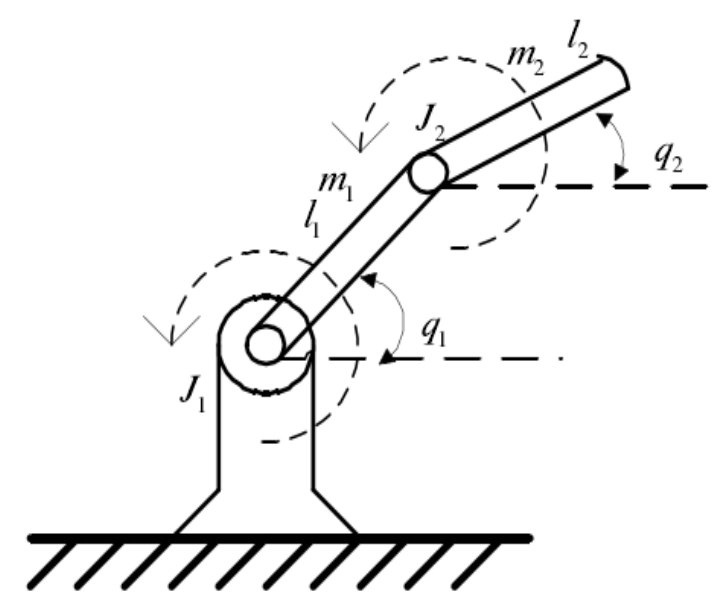

Fig. 2. Sketch map of a 2-degree-of-freedom robot

In Fig. $2 l_{1}, m_{1}$ and $q_{1}$ respectively denote the length, quality and corresponding angle of the robot connecting rod $1 ; l_{2}, m_{2}$ and $q_{2}$ are respectively the length, quality and corresponding angle of the robot connecting rod 2. All the above parameters cannot be measured accurately and are uncertain.

In the process of simulation, set $q=\left[\begin{array}{ll}q_{1} & q_{2}\end{array}\right]^{\mathrm{T}}, \tau=\left[\begin{array}{ll}\tau_{1} & \tau_{2}\end{array}\right]^{\mathrm{T}}$.

The upper limits of the robot dynamic friction force and the static friction force are respectively:

$$
\gamma_{\mathrm{s}}=\left(\begin{array}{ll}
0.05 & 0.05
\end{array}\right)^{\mathrm{T}}, \gamma_{\mathrm{d}}=\left(\begin{array}{ll}
0.05 & 0.05
\end{array}\right)^{\mathrm{T}}, \varepsilon=0.02 .
$$

The interference signal of the robots is $d(t)=\left[\begin{array}{ll}\sin t & \sin t\end{array}\right]$.

For the mode of compensation $n_{z}=2 ; A_{z}=0 I ; \quad B_{z}=I$; $C_{z}=200 I ; D_{z}=20 I$. The original state is 0 . Taking $Q=\operatorname{diag}[1000,100]$, by (7) we can get $P$.

The position instructions of two joints are respectively $q_{1 d}=0.5 \cos (\pi t)$ and $q_{2 d}=0.5 \sin (\pi t)$. The input of the neural network is the desired trajectory; the initial weight value is set to 0 , and the state of the system is $x=\left[\begin{array}{ll}q_{1} & \dot{q}_{1} \\ q_{2} & \dot{q}_{2}\end{array}\right]^{\mathrm{T}}$; the initial state is $\left[\begin{array}{llll}0 & 0.2 & -0.25 & 0.1\end{array}\right]^{\mathrm{T}}$. 
The response control parameters are set to: $F_{r}=\operatorname{diag}[10,6]$, $F_{x}=[120,1250 ; 12,125], \quad \gamma_{5}=50, \quad K_{d}=\varepsilon_{M}+d_{M}, \quad K_{\tau}=\operatorname{diag}[60,8]$, $\Gamma=\operatorname{diag}(20,20), \Gamma^{\prime}=\operatorname{diag}(0.12,0.13), \varepsilon_{1}=1, \varepsilon_{2}=0.5, \varphi_{\max }=10$.

The simulation results can be obtained as follows.
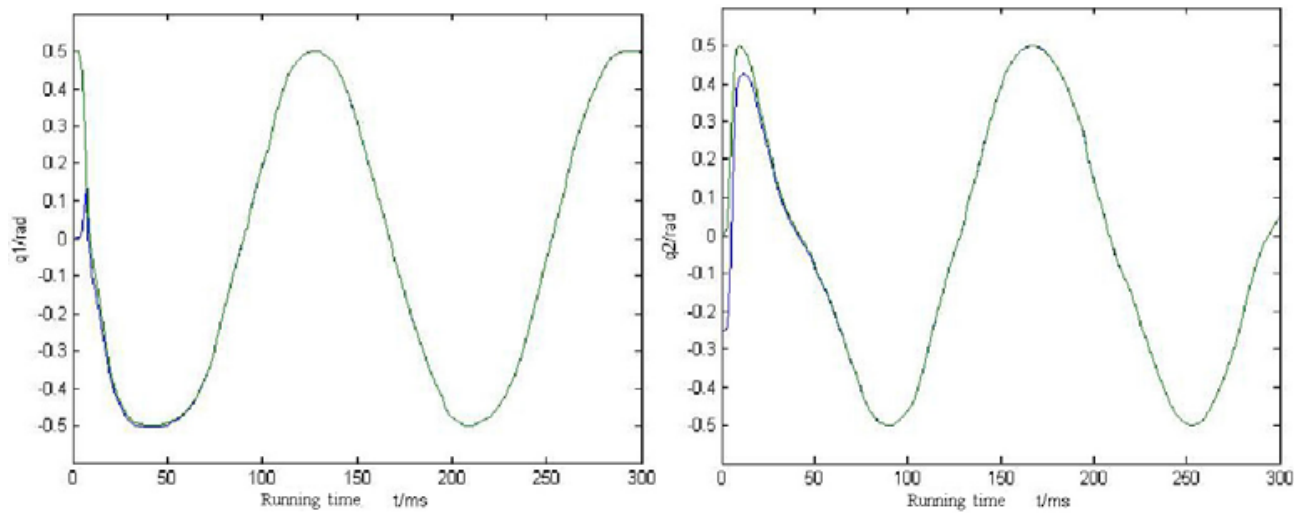

Fig. 3. Tracking curves of two links using this controller
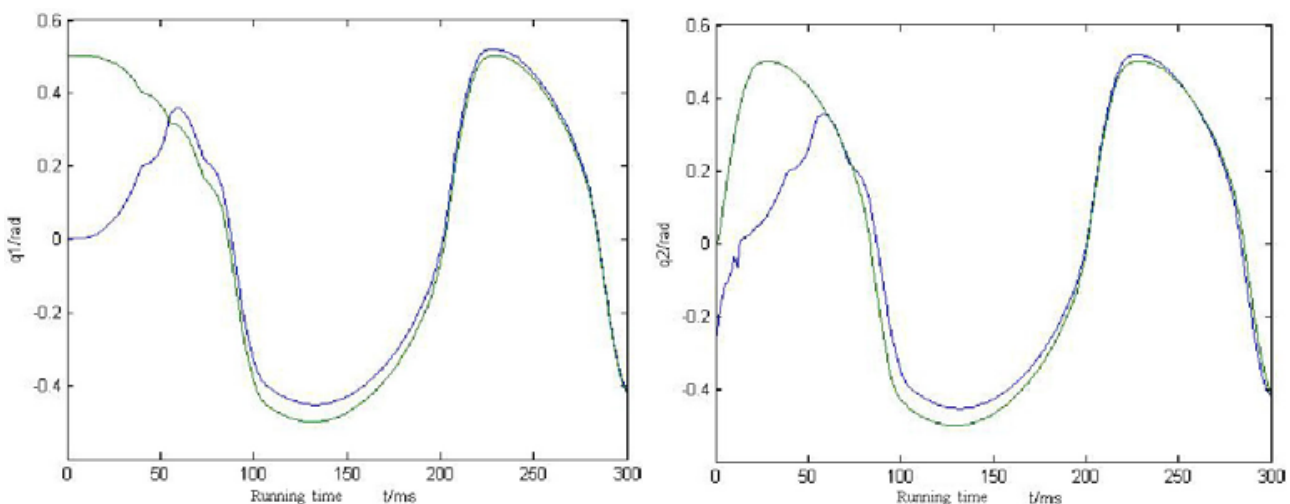

Fig. 4. Tracking curves of two links using adaptive control methods
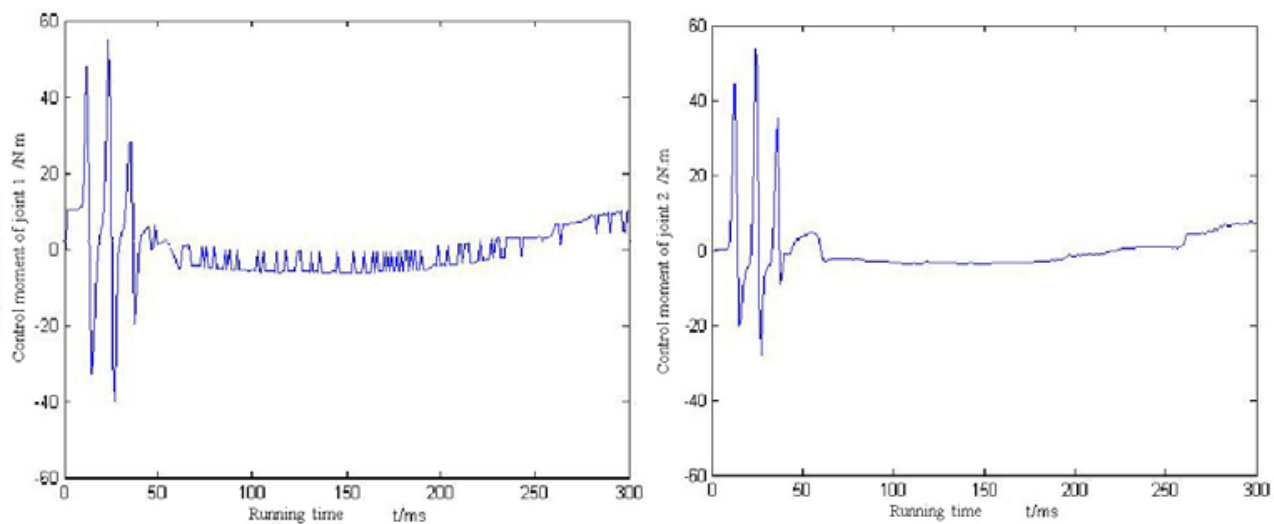

Fig. 5. Control input of both joints 
Based on Figs 3 and 4 we can see that under the circumstance that there is a nonlinear friction, external disturbance and unclear dynamic model items in the robot system, using a robust adaptive controller, based on the desired trajectory compensation can guarantee the actual trajectory track the desired trajectory faster and more efficiently. At the same time it ensures the system stability compared to a robust adaptive controller [18].The system has strong robustness. Fig. 5 shows the introduction of the continuous control rate, that efficiently avoids the chattering problem in the traditional variable structure control.

\section{Conclusion}

This paper studies a kind of a robust adaptive control method based on neural networks for robots. It does not need off-line calculation of the linear regression matrix of the system. Neural networks are used to identify the nominal model of the robot. The input signals of the neural networks are the desired trajectories of each joint, and the desired trajectories are determined and bounded, which eliminates the strict assumption of the control input in conventional neural networks. Besides, model compensation and a variable structure control rate are introduced to ensure the global exponentially asymptotic stability of the closed-loop system. It guarantees good transient performance and efficiently eliminates the chattering of the control moments. At the end, the simulation results show that the algorithm has very strong robustness and applicability.

Acknowledgement: The paper is sponsored by the Provincial University Natural Science Research Foundation of Jiangsu Education Department (12KJD510006, 13KJD520004, 14KJB520012); Natural Science Foundation of Jiangsu (BK2012082).

\section{References}

1. F a t e h, M. M., A. A z a r far. Improving Fuzzy Control of Robot Manipulators by Increasing Scaling Factors. Innovative Computing. - Information and Control-Express Letters, Vol. 3, 2010, No 3, 513-518.

2. C a li s e, A. J., N. Hov a k i m y a n, M. I d a n. Adaptive Output Feedback Control of Nonlinear Systems Using Neural Networks. - Automatica, Vol. 37, 2001, No 8, 1201-1211.

3. D a F e i p e n g. Decentralized Sliding Mode Adaptive Controller Design Based on Fuzzy Neural Networks for Interconnected Uncertain Nonlinear Systems. - IEEE Transactions on Neural Networks, Vol. 11, 2000, No 6, 1471-1480.

4. Suresh, S., N. Kannan. Direct Adaptive Neural Flight Control System for an Unstable Unmanned Aircraft. - Applied Soft Computing, Vol. 8, 2008, No 2, 937-948.

5. H o, D. W. C., J. L i, Y. N i u. Adaptive Neural Control for a Class of Nonlinearly Parametric Time-Delay Systems. - IEEE Transactions on Neural Network, Vol. 16, 2005, No 3, $625-635$

6. K a raka s og l u, A. A Recurrent Neural Network Based Adaptive Variable Structure ModelFollowing Control of Robotic Manipulator. - Automatica, Vol. 31, 1995, No 10, 1495-1507.

7. Hu ang, Guang-B in, S a r a t c handran, $\mathrm{N}$ ar a si m h a n S und a rarajan. An Efficient Sequential Learning Algorithm for Growing and Pruning RBF(GAP-RBF) Networks. - IEEE Transaction on Systems, Man, and Cybernetics - Part B: Cybernetics, Vol. 34, 2004, No 6, 2284-2292. 
8. Huang, Guang-Bin, Saratchandran, Narasimhan Sundararajan. A Generalized Growing and Pruning RBF (GGP-RBF) Neural Network for Function Approximation. - Control Theory and Applications, Vol. 16, 2005, No 1, 57-67.

9. H o r n g, J. H., J. G. H s i s e h. Adaptive Tracking Control of a Class of Nonlinear Systems Using CMAC Neural Network [J]. - Journal of Franklin Institute, Vol. 333B, 1996, No 6, 861-878.

10. L i a n li u, Z., J. S v o b od a. A New Control Scheme for Nonlinear Systems with Disturbances. - IEEE Transactions on Control Systems Technology, Vol. 14, 2006, No 1, 176-181.

11. Mou, Chen, Jiang Changsheng, Wu Qingxian. A Kind of Uncertain Nonlinear System Robust $\mathrm{H}_{\infty}$ Control Based on the Disturbance Observer. - Control Theory and Applications, Vol. 23, 2006, No 4, 611-614.

12. Ch e n, Y., J. L. Chang. Sliding-Mode Force Control of Manipulators. - In: Proceedings of National Science Council, Vol. 23, 1999, 281-289.

13. W e nhui, Zhang, Q i N a i ming, Y in Hongliang. Robot Trajectory Tracking Control of Adaptive Neural Variable Structure. - Control and Decision Making, Vol. 26, 2011, No 4, 597-600.

14. Yao, B., M. Tomizuka. Smooth Robust Adaptive Sliding Mode Control of Robot Manipulators with Guaranteed Transient Performance. - Journal of Dynamic Systems, Measurement and Control, Vol. 29, 1993, No 1, 1176-1180.

15. S u n, F. C., Z. Q. S u n. Stable Neuro-Adaptive Control of Robots with the Upper Bound Estimated on the Neural Approximation Errors. - Journal of Intelligent and Robotic Systems, Vol. 26, 1999, No 1, 91-100.

16. Ze in ali, M., L. Notash. Adaptive Sliding Mode Control with Uncertainty Estimator for Robot Manipulators. - Mechanism and Machine Theory, Vol. 45, 2010, No 1, 80-90.

17. Jin le i, Zhou, Zhang Weihai. The Robot Robust Control with Friction. - Journal of Mechanical Engineering, Vol. 43, 2007, No 9, 102-106.

18. Li, S hij in, J i e fa W a n g, Z ure n F e n. The Simulation Analysis of Robot System Based on Adaptive Variable Structure Control. - Journal of System Simulation, Vol. 14, 2002, No $9,1255-1258$. 